\title{
Formatos de publicidad no convencional en televisión versus spots: un análisis basado en el recuerdo
}

\author{
Eva REINARES-LARA \\ Universidad Rey Juan Carlos \\ eva.reinares@urjc.es \\ Pedro REINARES-LARA \\ Universidad Rey Juan Carlos \\ pedro.reinaresurjc.es \\ Cristina Olarte-PASCUAL \\ Universidad de La Rioja \\ cristina.olarteunirioja.es
}

Recibido: 20 de noviembre de 2015

Aceptado: 19 de abril de 2016

\begin{abstract}
Resumen
Las cifras de inversión publicitaria y audiencia indican el continuo liderazgo de la televisión como medio masivo. Sin embargo problemas como el zapping, la saturación publicitaria o la fragmentación de audiencias cuestionan su eficacia como medio de comunicación comercial. Este contexto ha propiciado el desarrollo de formatos de publicidad no convencional en televisión. Con el objetivo de proporcionar las necesarias evidencias empíricas se analiza, en un entorno real de audiencia, el recuerdo que generan cuatro formatos de publicidad no convencional: microprogramas, patrocinio televisivo, telepromoción interna y telepromoción externa, frente a la publicidad más convencional, el spot. La metodología empleada ha integrado de forma conjunta datos secundarios de audimetría y datos primarios procedentes de una encuesta ad-hoc telefónica aplicada a una muestra de 2.000 individuos de 16 a 65 años, representativos del total de la audiencia de televisión. Los resultados muestran la eficacia a nivel cognitivo de las formas no convencionales de publicidad ya que proporcionan mayores niveles de recuerdo espontáneo $\mathrm{y}$ asistido.
\end{abstract}

Palabras clave: Publicidad no convencional; nuevas formas de publicidad en televisión; spots, publicidad en televisión; eficacia publicitaria; recuerdo publicitario.

\section{Non-conventional advertising formats in television versus spots: An analysis based on the generated recall}

\begin{abstract}
Advertising investment and audience figures indicate that television continues to lead as a mass advertising medium. However, its effectiveness is questioned due to problems such as zapping, saturation and audience fragmentation. This has favoured the development of non-conventional advertising formats. This study provides empirical evidence for the theoretical development. This investigation analyzes the recall generated by four non-conventional advertising formats in a real environment: short programme (branded content), television sponsorship, internal and external telepromotion versus the more conven-
\end{abstract}


tional spot. The methodology employed has integrated secondary data with primary data from computer assisted telephone interviewing (CATI) were performed $a d-h o c$ on a sample of 2000 individuals, aged 16 to 65 , representative of the total television audience. Our findings show that non-conventional advertising formats are more effective at a cognitive level, as they generate higher levels of both unaided and aided recall, in all analyzed formats when compared to the spot.

Keywords: Non-conventional television advertising; new forms of television advertising; spots; television advertising; advertising effectiveness; advertising recall.

\section{Referencia normalizada}

Reinares-Lara, E., Reinares-Lara, P. y Olarte-Pascual, C. (2016). Formatos de publicidad no convencional en televisión versus spots: un análisis basado en el recuerdo. Historia y Comunicación Social. Vol 21, número 1, páginas 257-278.

Sumario: 1. Introducción. 2. Conceptualización de las nuevas formas publicitarias. 3. Hipótesis de la investigación. 3.1. Telepromoción interna. 3.2. Telepromoción externa. 3.3. Patrocinio televisivo. 3.4. Microprogramas con contenido publicitario. 4. Metodología. 5. Análisis de resultados. 6. Conclusiones.. 7. Referencias bibliográficas.

\section{Introducción}

La televisión destaca sobre el conjunto de medios como observatorio de las tendencias y perspectivas del negocio publicitario en general. Sus cualidades como medio publicitario están bien documentadas (Rubinson, 2009). Diferentes estudios afirman que la televisión continuará siendo el soporte publicitario mayoritario, a pesar de las limitaciones intrínsecas procedentes del actual entorno competitivo (Lowrey et al., 2005) relacionadas con: 1) la fragmentación de la audiencia (Chowdhury et al., 2007; Sharp et al., 2009), 2) el zapping (Tse y Lee, 2001), y, 3) la saturación publicitaria (Reinares y Reinares, 2007).

La aparente pérdida de eficacia relacionada con estas limitaciones (Smit et al., 2009), exige una utilización más creativa del medio para conseguir notoriedad y recuerdo publicitario (Dawson, 1996). En este contexto, la publicidad televisiva se está desarrollando más allá de los cortes publicitarios convencionales: se redefinen los contenidos de los programas y los contenidos comerciales se integran en los mismos (Farré y Fernández, 2005; Tur Viñes y Segarra Saavedra, 2013). Este hecho exige considerar cómo se está combinando la publicidad y el contenido de los programas para ampliar las opciones creativas y la presencia de las marcas en la pantalla a través de nuevas formas publicitarias (Hackley y Tiwsakul, 2006).

Tanto en el sector empresarial como en el académico se utilizan diversos términos para referirse a este conjunto de formatos publicitarios, tales como "publicidad especial", "nuevos formatos de publicidad en televisión" (Martín et al., 2012) o "brand placement" (Smit et al., 2009). En este trabajo se utiliza para denominarlos el término Formas Publicitarias No Convencionales (FNC). En él se integran todas las formas de 
publicidad en televisión diferentes formalmente de los spots convencionales (Woods, 2008; Tur Viñes y Segarra Saavedra, 2013).

La competencia actual entre medios y soportes publicitarios hace que las cadenas de televisión, con modelos de negocio basados en los ingresos publicitarios, se vean obligadas a innovar en la optimización del espacio publicitario. En este contexto, las FNC tienen una importancia estratégica y se han convertido en una fuente de ingresos fundamental para las televisiones generalistas (Arrazola et al., 2013).

Con el aumento significativo de alternativas a los spots de 20 segundos y las crecientes posibilidades comerciales de los nuevos canales de televisión, hay una demanda sin precedente en los departamentos comerciales de las cadenas para incorporar en su oferta comercial nuevas formas de publicidad. Los anunciantes consideran el fenómeno como una interesante forma de promover sus marcas más allá de la publicidad convencional basada en spots (Smit et al., 2009). De hecho, investigadores como Lowrey et al. (2005), ven en las alternativas a los spots convencionales "el futuro de la publicidad", dado el entorno tecnológico que favorece que las audiencias puedan evitar los mensajes comerciales. Para explicar su creciente desarrollo, varios "case studies" e investigaciones han demostrado que la publicidad no convencional genera más atención y recuerdo en las audiencias que los spots (Schmid, 2003; Media Planning Group, 2007; Reinares y Reinares, 2009; Arrazola et al., 2013; Reinares y Reinares, 2011; Martín et al., 2012). Al margen de estos trabajos, gran parte de las afirmaciones sobre su mayor capacidad para generar recuerdo, se han basado en la valoración de sus gestores (IP Network, 2007), con la credibilidad que dan las prácticas profesionales, pero sin el rigor necesario en la investigación académica.

La cualidad de generar mayor recuerdo de las FNC explica que los anunciantes hayan adoptado las mismas de forma generalizada en la planificación del medio televisión. Sin embargo, el dinamismo de las prácticas publicitarias en televisión tiene importantes carencias sobre aspectos básicos de su ejecución, lo que impide optimizar su rendimiento. En el contexto de la consolidación de las formas no convencionales en las estrategias publicitarias que usan el medio televisión, parece conveniente promover una investigación que aporte conocimiento a las prácticas de gestión desarrolladas actualmente por las cadenas (Walliser, 2003).

Respecto a los indicadores de eficacia propuestos en la investigación, el recuerdo de marca es uno de los objetivos más comunes buscado por los publicistas cuando planifican acciones de publicidad convencional en el medio televisión (Rothschild y Churchill, 1988). Sin embargo, como se ha señalado, no se disponen de trabajos relevantes que, con metodología científica, analicen de forma conjunta el recuerdo que generan las diferentes tipologías de publicidad utilizadas actualmente en televisión. Por ello, en este trabajo, se pretende realizar una investigación, en un entorno real, que permita analizar la eficacia comparada de los nuevos formatos de publicidad en televisión en relación a los spots, de acuerdo a objetivos publicitarios cognitivos, y aportar las necesarias evidencias empíricas para su correcta planificación. 


\section{Conceptualización de las nuevas formas publicitarias}

Al igual que en su nomenclatura, no hay consenso respecto a la conceptualización de las FNC, aunque varios autores han propuesto distintas definiciones y categorías de las mismas. Hackley y Tiwsakul (2006) introducen el término "entertainment marketing", que integra cualquier forma de presentar la marca dentro de contenido propio del medio televisivo. La práctica del entertainment marketing crea una relación simbiótica entre la comunicación persuasiva y los programas. Esta conceptualización de las FNC a partir de la integración de los mensajes comerciales con los contenidos televisivos se recoge en fuentes posteriores (Smit et al., 2009).

En la actualidad, se pueden identificar una gran variedad de tipologías publicitarias en televisión con características formales, gestión y objetivos comunicacionales distintos del spot convencional. El spot se integra en los espacios publicitarios de forma independiente, ya que mantiene su monopolio enunciativo al margen del canal y se difunde de forma reiterada a través de diferentes soportes en el marco de una campaña. Mientras que las FNC aportan un valor añadido en su capacidad de persuasión, integrándose de diferentes formas junto a los programas y desarrollando su creatividad al aprovechar las cualidades del espacio que anteceden o preceden.

Las diferentes integraciones de los elementos comerciales en los contenidos del medio dan lugar a las distintas tipologías de FNC ofertadas por las cadenas. Estas opciones incluyen, entre otras, el patrocinio televisivo, publicidad en pantalla compartida, microprogramas, emplazamiento de producto, televenta, telepromociones, momentos internos o sobreimpresiones (Farré y Fernández, 2005).

Del conjunto de formas publicitarias en televisión diferentes al spot, identificadas a partir de la observación de las prácticas habituales del mercado (Reinares y Reinares, 2007), se han seleccionado para la investigación cuatro tipologías que se comercializan en la mayor parte de cadenas generalistas en el ámbito internacional (Egta, 2008) con el objetivo de ampliar la validez de las aportaciones de este trabajo. Son formatos con un desarrollo comercial consolidado en el tiempo (al menos 10 años) y que presentan unas características formales diferenciadas. Ello permite una definición precisa y estable de cada formato y se facilita su identificación dentro de las emisiones. Otro criterio considerado en la selección de los formatos de estudio ha sido el requisito de que, en la suma de sus características, quede recogido el conjunto de cualidades que definen las FNC, de manera que los resultados permitan hacer inferencias, además de a cada formato analizado, también al conjunto de FNC en uso.

En el Cuadro 1 se caracterizan los cuatro formatos no convencionales analizados en este trabajo (Reinares y Reinares, 2003): 
Cuadro 1. Formas no convencionales investigadas

\begin{tabular}{|c|c|c|}
\hline & $\begin{array}{l}\text { Microprogramas con contenido } \\
\text { publicitario }\end{array}$ & Patrocinio televisivo \\
\hline- & $\begin{array}{l}\text { Programa de 1-2 minutos patrocinado } \\
\text { por el anunciante con contenidos } \\
\text { agrupados en torno a un eje temático. } \\
\text { Adoptan un tono intermedio entre } \\
\text { lo divulgativo y lo informativo, con } \\
\text { el objetivo de despertar el interés de } \\
\text { perfiles de audiencia homogéneos y, } \\
\text { por lo general, difíciles de impactar. }\end{array}$ & $\begin{array}{l}\text { Piezas de un máximo de } 10 \text { segundos } \\
\text { emitidas inmediatamente antes o después } \\
\text { del programa patrocinado (incluidos } \\
\text { los bloques publicitarios intermedios } \\
\text { del programa, donde se planifican en } \\
\text { última posición; pegados, por tanto, al } \\
\text { programa), en las que se comunica quién } \\
\text { es el patrocinador del programa mientras } \\
\text { se realiza publicidad del mismo. } \\
\text { - Su propósito es vincularla marca con el } \\
\text { programa patrocinado para beneficiarse } \\
\text { de las asociaciones positivas entre } \\
\text { ambos. }\end{array}$ \\
\hline & Telepromoción externa & $\begin{array}{l}\text { Telepromoción interna } \\
\end{array}$ \\
\hline & $\begin{array}{l}\text { Piezas de al menos } 60 \text { segundos, } \\
\text { emitidas en posición preferente en } \\
\text { el bloque publicitario, en las que } \\
\text { personas y personajes de una cadena } \\
\text { realizan la comunicación comercial } \\
\text { del anunciante a partir de un desarrollo } \\
\text { creativo inherente al papel habitual de } \\
\text { ese personaje en la cadena. } \\
\text { Persiguen una asociación con } \\
\text { el programa al que se vinculan } \\
\text { creativamente, de manera que se } \\
\text { obtenga un mayor impacto sobre la } \\
\text { audiencia del programa y, con ello, del } \\
\text { público objetivo del anunciante. }\end{array}$ & $\begin{array}{l}\text { Comunicaciones comerciales de } \\
\text { mayor duración que un spot, realizadas } \\
\text { dentro de un programa por el/los } \\
\text { conductor/es habituales, que asumen } \\
\text { el rol de prescriptores de los productos } \\
\text { presentados. } \\
\text { - El objetivo es vincular el producto a las } \\
\text { caras más conocidas de un canal para } \\
\text { propiciar asociaciones. La integración } \\
\text { con los programas es alta, puesto que } \\
\text { al ir dentro del programa, no hay fuga } \\
\text { publicitaria por el zapping. El anunciante } \\
\text { puede seleccionar el público objetivo a } \\
\text { impactar en función del perfil de cada } \\
\text { programa. }\end{array}$ \\
\hline
\end{tabular}

\section{Hipótesis de la investigación}

A partir del objetivo genérico planteado en la introducción, se hace necesario proponer un conjunto de hipótesis que permitan contrastar las capacidades cognoscitivas de las diferentes FNC estudiadas frente a los spots. Para su formulación existen escasos antecedentes teóricos en la literatura centrados, de forma específica, en los formatos analizados en este trabajo. Sin embargo, considerando sus características 
diferenciales frente a los spots, la literatura genérica sobre eficacia publicitaria en televisión resulta un antecedente válido que permite inferir, para las cuatro tipologías objeto de estudio, una mayor capacidad para generar recuerdo.

\subsection{Telepromoción interna}

Dado que su elemento diferencial es que son mensajes comerciales explícitos que se integran dentro de los contenidos televisivos no publicitarios (programas), es viable relacionar su eficacia cognitiva con el llamado "efecto sorpresa" propuesto, entre otros, por Russell (2002) o Lee y Faber (2007), derivado de su ubicación fuera del entorno habitual de los mensajes publicitarios (bloque publicitario). A este respecto Balasubramanian (1994) afirma que el mayor recuerdo de las FNC se origina por el efecto sorpresa que generan en las audiencias al no esperar encontrarse con mensajes comerciales dentro de los programas. La teoría contextual propone que aquellos elementos que contrastan con su entorno destacarán más, y, por lo tanto, tendrán más posibilidades de ser recordados que los de su propio contexto (Baddeley, 1990).

Otro antecedente teórico que puede anticipar este mayor recuerdo es el hecho de que el anuncio convencional aparece en un bloque publicitario junto con otros anuncios, siendo por ello afectado negativamente en el recuerdo (Pieters y Bijmolt, 1997), frente a la nula competencia de mensajes comerciales que la telepromoción interna tiene en el programa.

Otros factores moderadores del recuerdo estudiados en la literatura académica han sido el tipo de programa con el que se integra el formato (Furnham et al., 2002; Bartel-Sheehan y Guo, 2005), la congruencia entre el formato y el programa (Lord y Burnkrant, 1993), o la utilización de personajes famosos en el formato (Amos et al., 2008). Todos estos factores podrían anticipar un mayor recuerdo a favor de la telepromoción interna como se prepone en la primera Hipótesis:

H1. La telepromoción interna genera un mayor recuerdo que los spots.

\subsection{Telepromoción externa}

Como se ha señalado en su descripción, formalmente se caracteriza por ser una forma publicitaria basada en una creatividad que asocia los mensajes comerciales con los contenidos televisivos (de forma similar a la telepromoción interna) junto con una posición privilegiada dentro del bloque publicitario (primera o última posición). Por lo tanto cabe explicar su eventual mayor capacidad para ser recordada desde estas dos características.

A partir de las propuestas de autores como Hetsroni y Asya (2002), Lowrey (2006), Moorman et al. (2007) o Smit et al. (2009) se puede inferir que la asociación que la audiencia hace de los contenidos de la telepromoción externa con un programa de entretenimiento, y los efectos positivos derivados de esta integración, puede ser la justificación que explica que se recuerden más que los spots. Por otra parte, parece asumido por la investigación académica (Newell et al., 2001; Scott, 2005) que los 
mensajes comerciales colocados en primera y última posición, dentro de un bloque publicitario, son mejor recordados que aquellos que están colocados en las posiciones intermedias. El efecto primacía también ha sido contrastado por autores como Moorman et al. (2007), Gupta y Gould (2007) o, Li (2010), que concreta el efecto posición preferencial (primacía) hacia el recuerdo en un trabajo sobre spots. Este efecto puede explicarse sobre la base de dos antecedentes teóricos. Por una parte, el proceso de interferencia entre los diferentes mensajes contribuye a explicar la primacía y la recencia en el recuerdo de los anuncios en televisión (Burke y Srull, 1988). Y, por otra parte, de acuerdo con las teorías del procesamiento de la información, cuantos menos mensajes se presentan a los espectadores, mayor es la propensión a prestar atención y la probabilidad de procesar la información a un nivel más profundo (Ha y McCann, 2008).

Dado estos antecedentes relacionados con sus características formales distintivas se puede justificar la siguiente Hipótesis:

H2. La telepromoción externa genera un mayor recuerdo que los spots.

\subsection{Patrocinio televisivo}

Quizás por su mayor afinidad formal con el spot, es uno de los formatos más analizados. Salvo algunas excepciones, como el trabajo de Tiwsakul et al. (2005), en el que no se encuentran relaciones positivas en el recuerdo generado de la marca tras su integración en un patrocinio televisivo, el resto de trabajos encuentran una relación positiva entre su uso, condicionado por determinados factores de influencia, y los efectos cognitivos en las audiencias (Meir et al., 1997, Lardinoit, 1999; Mcdaniel, 1999).

De los argumentos que explicarían el mayor recuerdo se puede destacar que el patrocinio presenta la cualidad formal diferencial de ser un formato publicitario corto. En un entorno tan saturado de publicidad, esta cualidad puede ser decisiva para aminorar el rechazo de las audiencias hacia los mensajes comerciales, lo que se trasladaría en un mayor recuerdo en relación a otros formatos considerados más largos (Stanton y Burke, 1998). También, a partir de los trabajos de Lowrey (2006) y Romaniuk (2009), en los que se pone de manifiesto que formatos con menor duración generan un mayor aprendizaje en los telespectadores, se puede inferir que, el patrocinio presentaría una relación más favorable de aprendizaje de los contenidos, dado que por sus características formales, los mensajes contenidos en 10 segundos, deben ser necesariamente más reducidos que los que se pueden trasladar en formatos de mayor duración, como el spot, las telepromociones o los microprogramas publicitarios.

En el contexto de los anteriores argumentos, Jeong et al. (2011), llevan a cabo un estudio comparativo entre spots y patrocinio televisivo, donde contrastan que la saturación de cortes publicitarios que integran un gran número de spots, conlleva una inhibición en los espectadores para recordar la información, dando lugar a una menor atención y mayor confusión. Sin embargo esta relación negativa no se contrasta en el patrocinio televisivo. Las posibles explicaciones que el autor considera se refieren 
a la naturaleza formal del patrocinio, que pueden dar lugar a que no se perciba su carácter comercial y se visione con la misma atención prestada a los programas.

Por lo tanto la Hipótesis 3 se formula con el siguiente sentido:

H3. El patrocinio genera un mayor recuerdo que los spots.

\subsection{Microprogramas con contenido publicitario}

En esta FNC los mensajes comerciales aparecen completamente integrados en los contenidos del programa. Se diseñan para entretener a las audiencias, por lo que se evitan referencias comerciales explicitas propias del resto de formatos publicitarios. Precisamente el hecho de que la audiencia los perciba como programas, y por lo tanto los visione por su capacidad de entretenimiento, permitiría explicar el mayor recuerdo que generan. Según antecedentes teóricos propuestos por Hackley y Tiwsakul (2006), Masterson (2005) o Chowdhury et al. (2007), la capacidad de entretenimiento de la FNC genera interés hacia el formato en sí mismo. De esta manera se puede explicar que los mensajes comerciales contenidos en los microprogramas sean mejor recordados por su capacidad para captar la atención que los que se trasladan a las audiencias en forma de spots.

En esta línea, Gupta y Gould, (2007) argumentan que, cuando los mensajes comerciales se integran en los programas, provocan menos "reactancia" en las audiencias que los spots convencionales. De acuerdo con Dahlén y Edenius (2007) esto se puede justificar porque, cuando las audiencias conocen el carácter persuasivo de los mensajes, establecen mecanismos de protección frente a ellos.

Por lo tanto parece coherente contrastar que:

H4. El microprograma con contenido publicitario genera mayor recuerdo que los spots.

\section{Metodología}

España representa el mercado televisivo idóneo para la aplicación de esta investigación, tanto por la importancia de la publicidad en el medio como por el mayor desarrollo de estas formas de publicidad frente a otros mercados televisivos (Carat, 2005; Arrazola el al., 2013).

En el presente trabajo se propone la medición de la eficacia publicitaria con técnicas basadas en la memoria. Del conjunto de técnicas de carácter cognoscitivo, el recuerdo se utiliza como una medida de la capacidad de un anuncio para generar la atención y atraer el interés (Ross, 1982) y, a lo largo de la investigación publicitaria, ha sido considerado como una medida relativamente válida y fiable en este propósito (véase Rothschild y Churchill, 1998; Romaniuk y Wight, 2009; Jeong et al., 2011). Su utilización en la investigación sobre eficacia se fundamenta en la creencia de que la publicidad es más eficaz en la medida en que genera un mayor recuerdo, ya que 
en estas circunstancias es mayor la probabilidad de que se produzca un cambio de actitud y se consiga la conducta deseada (Beerli y Martín, 1998; Vakratsas y Ambler, 1999).

Para medir el nivel de aprendizaje que generan los mensajes comerciales, las fuentes consultadas utilizan, de forma generalizada, tanto el recuerdo espontáneo (unaided recall) como el recuerdo asistido (aided recall) (Furnham et al., 2002). Beerli y Martín (1998) consideran que el recuerdo asistido es una medida de eficacia más sensible y, por lo tanto, más fiable para detectar si el anuncio ha dejado alguna huella en la mente del individuo. Mientras que De Pelsmacker et al. (2005) señalan que el recuerdo espontáneo es una medida más fuerte de la memoria $\mathrm{y}$, por ello, más difícil de obtener frente al recuerdo sugerido. De esta manera, es frecuente encontrar trabajos que solo presentan resultados basados en el recuerdo asistido, dado los bajísimos niveles que genera el recuerdo publicitario espontáneo en investigaciones desarrolladas en un entorno real. Algunos autores en el ámbito académico, reportan la necesidad de utilizar simultáneamente el recuerdo espontáneo y el asistido para medir el efecto cognitivo de la publicidad (Moorman et al., 2007).

Con estas premisas, para evitar condicionar con la metodología aplicada los resultados de este trabajo, se propone un diseño de investigación que permite trabajar con ambos tipos de recuerdo, en la línea de trabajos como los de Pieters y Bijmolt (1997), y que ya ha sido aplicado satisfactoriamente en el trabajo de Arrazola et al. (2013).

Por su propia idiosincrasia, los nuevos formatos publicitarios tienen mayor audiencia que los spots al ir posicionados dentro de los programas o junto a ellos. Para comparar el recuerdo que generan a partir de una presión publicitaria equivalente, se hace necesario aislar la mayor audiencia de las nuevas formas publicitarias en relación a los spots. Para ello, se han integrado, por un lado los datos de audimetría de las empresa Kantar Media y, por otro, los datos del recuerdo publicitario procedentes de una encuesta ad-hoc telefónica (CATI). Este proceso ha sido necesario debido a que las encuestas telefónicas no permiten valorar el efecto directo de una sola inserción publicitaria, al no conocerse si el encuestado estaba delante del televisor en el momento concreto de la emisión. Combinando las dos fuentes se puede conocer fehacientemente la relación entre la presión publicitaria, la audiencia real que consigue cada formato publicitario, y sus efectos en términos de recuerdo para cada emisión de un estímulo publicitario, que en este caso son las diferentes formas de publicidad que contempla el estudio. Este proceso ha permitido crear un "coeficiente de ponderación" para cada una de las observaciones obtenidas, cuya aplicación posibilita aislar el efecto en el recuerdo, de las diferencias de audiencias alcanzadas por los formatos analizados. El coeficiente transforma la presión publicitaria de cada marca (GRP's) y su formato de publicidad, en impactos reales que generan recuerdo. Esta aportación metodológica tiene su origen en el hecho de que las tasas de recuerdo obtenidas mediante la aplicación de una encuesta no consideran el efecto de un anuncio en términos de niveles de audiencia real, sino sobre la base del tamaño muestral definido. De esta manera, las tasas de recuerdo publicitario no contemplan el impacto real que ha generado un anuncio, ya que, por ejemplo, si un anuncio ha obtenido 
una tasa de recuerdo del 10\% a partir de una encuesta, su tasa de recuerdo real será diferente en función de la audiencia real que haya tenido el bloque publicitario en el que se ha insertado el anuncio. Así, si la audiencia real ha sido del $80 \%$, una tasa de recuerdo del 10\% implica un menor impacto real en la audiencia que si estuviéramos ante una situación en la que la audiencia real fuese del $20 \%$. La aplicación de la metodología propuesta tiene como objetivo obtener unas tasas de recuerdo ajustadas a las audiencias reales de cada spot. El "coeficiente de ponderación" se aplica para cada anuncio, calculando el cociente entre el porcentaje de recuerdo espontáneo obtenido a través de la encuesta telefónica y el porcentaje de audiencia del bloque publicitario en que se ha emitido el spot publicitario. Este proceso también permite homogeneizar la duración de las diferentes formas de publicidad analizadas (oscilan entre 10 y 120 segundos), ya que como distintas fuentes han contrastado, existe hay una relación entre recuerdo y la duración del formato (véase Stanton y Burke, 1998).

Para la recogida de la información en las audiencias sobre el recuerdo espontáneo y asistido se utilizó, como se ha señalado, un cuestionario estructurado, diseñado ad-hoc y aplicado de forma personal por teléfono (CATI) a una muestra de 2.000 individuos de 16 a 65 años (véase Tabla 1). Se establecieron cuotas en función del género, edad, número de miembros de la unidad familiar y tamaño del municipio, al objeto de obtener una muestra fiable y representativa de la audiencia de televisión.

Tabla 1. Distribución de la muestra

\begin{tabular}{|llllll|}
\hline Género & $\%$ & Miembros de la unidad familiar & $\mathbf{\%}$ & Edad & $\%$ \\
Hombres & 39,2 & Hogares unipersonales & 16,7 & $16-19$ años & 12,9 \\
Mujeres & 60,8 & Hogares de 1 a 3 miembros & 32,6 & $20-24$ años & 10,9 \\
& & Hogares de 4 a 7 miembros & 41,5 & $25-34$ años & 15,4 \\
& & Hogares con más de 8 miembros & 9,2 & 35-44 años & 18,3 \\
& & & & $45-65$ años & 42,5 \\
\hline
\end{tabular}

La captación de los entrevistados se realizó dos días antes de la emisión de los programas mediante el sistema Random Digit Dialing. Todos los entrevistados daban su consentimiento para ser entrevistados a una hora determinada mientras estaban viendo la televisión en casa. Cada entrevistado fue interrogado por su visionado televisivo (programas contemplados) en los 30 minutos previos a la llamada (como mecanismo de control) y por las emisiones publicitarias que se emitieron en el cuarto de hora anterior al periodo horario en el que realizó la entrevista.

Respecto al cuestionario, éste se iniciaba con una pregunta de control que verificaba si el programa en el cual aparecía el bloque publicitario se había visto realmente. En la medición del recuerdo espontáneo se solicita la información al entrevistado sin proporcionarle ningún tipo de ayuda para estimular su recuerdo. En esta investigación se pidió a los entrevistados que mencionaran las marcas anunciadas en el bloque comercial estudiado, sin dar ninguna información de las mismas, a través de la pregunta "¿Qué anuncios de televisión y de qué marcas recuerdas haber visto en el canal X e Y durante el periodo Z? Por favor, indica todos los anuncios y marcas que 
recuerdas haber visto". Las respuestas se codificaron como correctas si el consumidor podía recordar tanto la categoría de producto como la marca. Mientras que en el recuerdo sugerido se ayuda al entrevistado recordándole ciertos elementos del anuncio. En la investigación se leyó al entrevistado una lista de marcas existentes en el mercado, de las cuales, solo la mitad había aparecido en el bloque objeto de estudio. A través de la pregunta "¿Cuáles de estas marcas has visto en el canal Xe Y durante el periodo Z?", los encuestados debían indicar si recordaban haber visto un anuncio publicitario de cada marca dentro del bloque específico de estudio.

El cuestionario se adaptó en cada entrevista mediante el sistema informático del Call-Center de forma automática a cada bloque/emisión publicitaria. Los elementos que se incorporaban a la entrevista en el momento de realizar la entrevista fueron: 1) el canal, 2) horario, 3) situación de emisión del corte publicitario y 4) marcas-productos que en el periodo testado habían emitido publicidad. Su duración final fue de 7 minutos como máximo.

De esta manera se ha obtenido un recuerdo real de la marca-producto (ej. Ford Focus C-Max) de todos aquellos spots y formatos no convencionales emitidos durante los meses de mayo y junio del año 2010 en el conjunto de cadenas generalistas de cobertura nacional que emiten en abierto en España. Ello ha supuesto la medición del recuerdo según los resultados de 41.487 observaciones (respuestas diferentes al recuerdo de anuncio publicitario, cada forma de comunicación valorada por un individuo que ha sido entrevistado se convierte en registro). Por tanto, si un individuo ha visto 30 anuncios, habrá dado lugar a 30 registros distintos por individuo-anuncio. Estas observaciones se reparten entre spots y las diferentes formas publicitarias no convencionales (FNC). Así la base de observaciones se distribuyó de la siguiente forma': Spots: 37.020; Formatos no convencionales: 4.467, repartidos de la siguiente forma: patrocinio 1.926; microprogramas 1.262; telepromoción externa $478 \mathrm{y}$, telepromoción interna 801 . Concretando la representatividad de marcas, categorías de productos y creatividades que proporciona el entorno real y la metodología de la investigación, la muestra recoge 1.512 spots y FNC diferentes, identificados a partir del sector de actividad y marca, abarcando 23 sectores y 450 marcas.

Desde un punto de vista metodológico, el valor de esta investigación se deriva de su realización en un entorno real, tal y como se recomienda en algunos trabajos como los de Pieters y Bijmolt (1997) y Moorman et al. (2007), ya que es imposible hacer generalizaciones sobre datos que no hayan sido obtenidos de esta forma. Estos autores consideran que en un laboratorio los recursos cognitivos se dedican exclusivamente a procesar la información de los programas y mensajes publicitarios a los que se expone a la muestra, planteando una situación alejada de las condiciones de audiencia de la vida real. 


\section{Análisis de resultados}

Los resultados de la investigación se pueden resumir en una afirmación: a igualdad de audiencia y duración, las nuevas formas de publicidad en televisión generan mayor recuerdo que la publicidad convencional (spots). Es destacable que estos resultados son congruentes con los escasos trabajos con objetivos similares centrados en el conjunto de FNC (Schmid, 2003; Media Planning Group, 2007; Arrazola et al., 2013).

El Gráfico 1 muestra que el recuerdo asistido es muy superior al recuerdo espontáneo. Todas las diferencias que se obtienen al comparar el recuerdo espontáneo de los spots y el conjunto de formas no convencionales y el recuerdo asistido de los spots y el conjunto de formas no convencionales son significativas ${ }^{2}$.

Gráfico 1. Indicadores de eficacia publicitaria en televisión por formatos (Porcentaje de recuerdo)

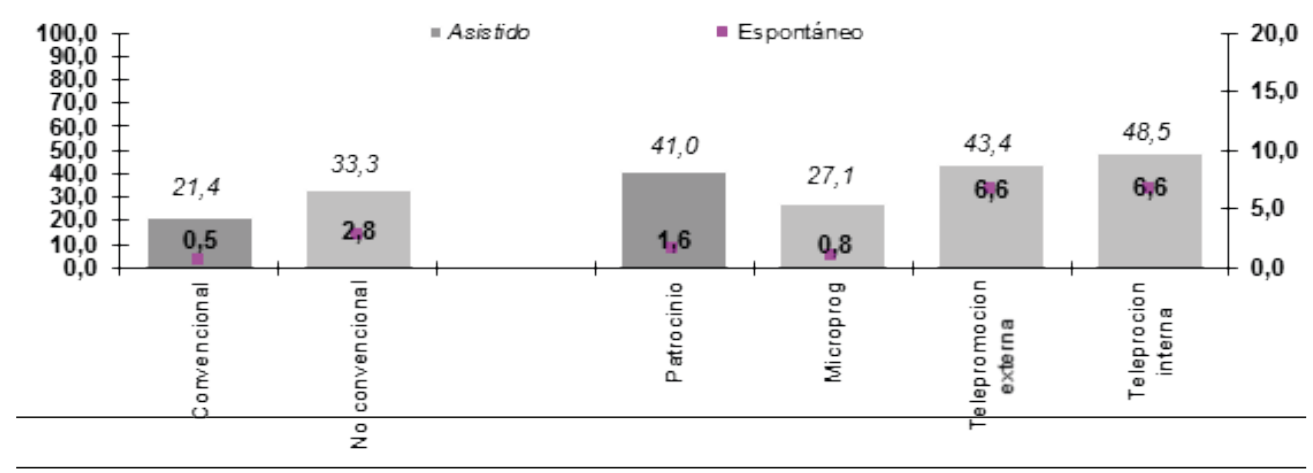

Como se puede observar, las cifras de recuerdo asistido oscilan entre el 21,4\% (spots) y el 48,5\% (telepromociones internas). Mientras que los valores del recuerdo espontáneo son mucho más bajos (entre $0,5 \%$ y $6,6 \%$ de los encuestados recuerdan haber visto el anuncio). Sin embargo estas diferencias entre los niveles máximo y mínimo presentan una mayor oscilación (12 veces) que la obtenida en los valores del recuerdo asistido (2 veces). Esto evidencia la conveniencia de utilizar tanto el recuerdo espontáneo como el asistido para valorar los efectos cognoscitivos, como se ha señalado en el epígrafe de metodología. Estas diferencias entre ambos tipos de recuerdo son coincidentes con trabajos que utilizan una metodología similar a la de este trabajo (Moorman et al., 2007).

Analizadas por separado, todas las tipologías no convencionales tienen individualmente mayor recuerdo asistido $\mathrm{y}^{3}$ espontáneo ${ }^{4}$ y que los spots. El orden de mayor a menor recuerdo asistido de los cuatro formatos estudiados es: $1^{\circ}$ ) telepromociones internas, $2^{\circ}$ ) telepromociones externas, $3^{\circ}$ ) patrocinios, $4^{\circ}$ ) microprogramas. Las telepromociones internas y externas general el mismo nivel de recuerdo espontaneo: 6,6 
entrevistados de cada 100 recuerdan haber visto estos dos formatos no convencionales.

Para una adecuada visualización de los resultados, se ha optado por presentar el recuerdo de los nuevos formatos en relación al recuerdo generado por spots. Para ello, se considera como base 100 el recuerdo que generan los spots. Considerando, por tanto, que ambos tipos de recuerdo (espontáneo y asistido), en los spots serían el índice base 100, obtenemos así los datos que presentan los gráficos 2 y 3 para los nuevos formatos, en su conjunto y para cada una de sus tipologías. Así, se inicia la descripción de los resultados presentando las diferencias que generan los spots y las nuevas formas publicitarias en el recuerdo espontáneo. Como muestra el Gráfico 2, se evidencia una mayor eficacia de los nuevos formatos con respecto a los spots. Estos generan en su conjunto un recuerdo espontáneo cinco veces superior a los spots (539 vs. 100).

Gráfico 2. Índice de recuerdo publicitario espontáneo por formatos (base 100 publicidad convencional)

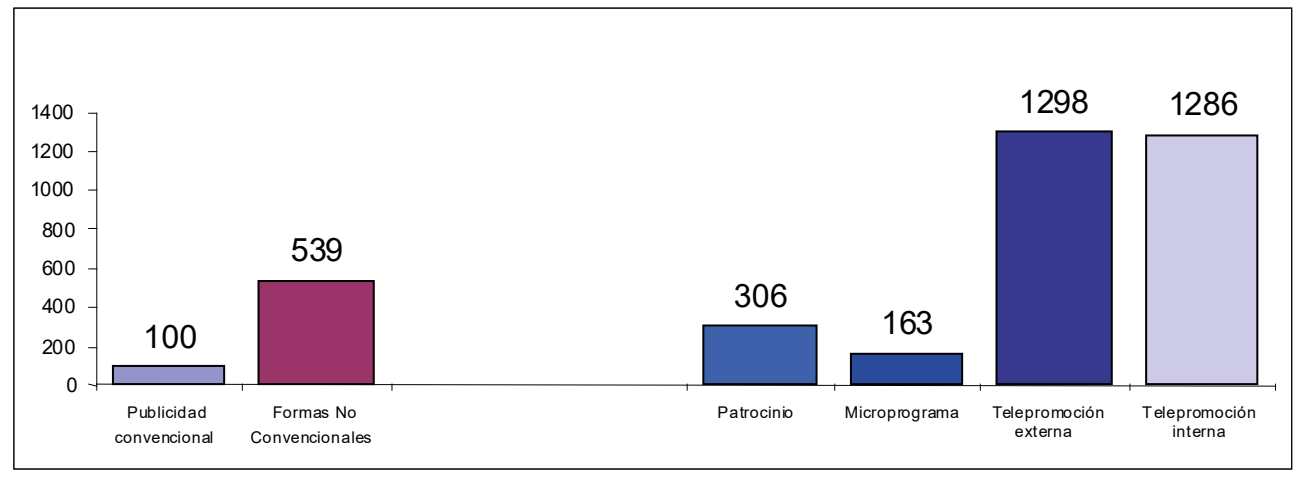

Analizadas por separado (Gráfico 2), todas las tipologías especiales tienen individualmente mayor recuerdo espontáneo que los spots. Un 66\% más en el caso de los microprogramas; el triple en el caso del patrocinio y casi trece veces más de recuerdo espontáneo en el caso de las telepromociones externas e internas.

Respecto a los resultados del recuerdo publicitario asistido (gráfico 3), se observa de nuevo, que las FNC generan mayor nivel de recuerdo que los spots; en concreto, un 56\% más. Este mayor recuerdo asistido varía en cada formato: un $27 \%$ más en el caso de los microprogramas; el torno al doble en el caso del patrocinio (92\%) y de las telepromociones (103\%); y un 127\% más en el caso de las telepromociones internas. 
Gráfico 3. Índice de recuerdo publicitario asistido por formatos (base 100 publicidad convencional)

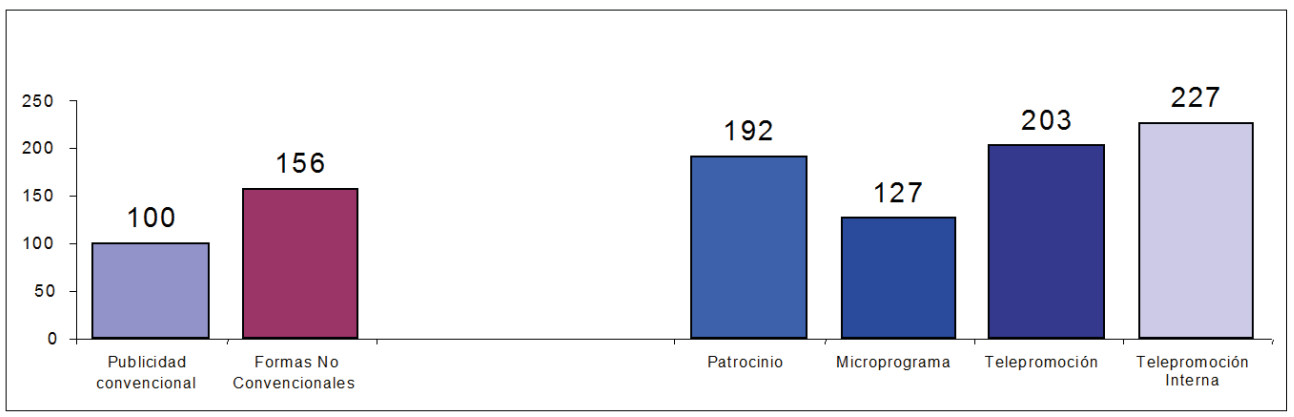

Se puede concluir, que el recuerdo espontáneo del conjunto de nuevas formas de publicidad, con respecto a los spots, es 3,5 veces superior al recuerdo asistido (539 versus 156). Por debajo de esa proporción se sitúan los patrocinios y los microprogramas, que aunque se muestran eficaces para generar elevados índices de recuerdo espontáneo, no respetan la proporción de 3,5 veces. Ambos formatos se muestran muy eficaces para generar recuerdo asistido y (tan solo) eficaces para generar recuerdo espontáneo. Por el contrario, dos formatos con gran capacidad de generar recuerdo espontáneo son las telepromociones internas y externas, que sobrepasan en ambos casos, la proporción media de incremento entre ambos tipos de recuerdo. Los dos formatos se revelan como los más eficaces a la hora de generar recuerdo espontáneo y asistido.

\section{Conclusiones}

Los resultados de esta investigación aportan las necesarias evidencias empíricas para el desarrollo teórico de los diferentes formatos no convencionales y proporcionan una guía operativa de gestión que permite la optimización del recuerdo que generan.

Pudiera parecer, a la vista de estos resultados a favor de las nuevas formas de publicidad, que es recomendable concentrar la presión publicitaria en televisión en estas tipologías, dado que se ha contrastado que son mucho más recordadas por el espectador. Incluso, se podría argumentar que, puesto que las telepromociones internas son el formato que más recuerdo genera, es viable concentrar la comunicación comercial en televisión en dicha forma publicitaria. Sin embargo conviene moderar estos resultados con otros elementos que faciliten la decisión de incorporar estas formas en los planes de comunicación. 
Ambas tipologías, spots y nuevos formatos, permiten satisfacer objetivos de comunicación bien definidos, y resultan útiles en las actuales estrategias de los anunciantes. Así, una campaña basada en spots, proporciona al anunciante frecuencia de impacto y cobertura, objetivos básicos en un plan de medios (Sharp et al., 2009). Como se ha descrito, las nuevas formas de publicidad proporcionan una mayor notoriedad, derivada de su mayor capacidad de generar recuerdo en la mente del espectador. Por tanto, se concluye que lo óptimo es la combinación de ambas en el plan de medios de televisión.

Igualmente, tampoco sería eficiente basar toda una campaña en telepromociones internas, por mucho que sean el formato publicitario que más recuerdo genera, dado que existen otros condicionantes formales, específicos en cada formato, que se deben considerar. Cada una de las tipologías tiene sus propios condicionantes operativos en cuanto a creatividad, duración, ubicación, complejidad en su producción, etc., que las hace completamente distintas entre sí; y que las puede hacer más o menos acordes a los objetivos de comunicación del anunciante. Todas las alternativas al spot generan mayores niveles de recuerdo. Sin embargo este resultado no las hace adecuadas para cualquier anunciante, campaña o estrategia publicitaria. Por ello es necesario considerar criterios cualitativos que no se suelen tener en cuenta cuando el anunciante elige estos formatos solo en función de su potencial recuerdo o de su coste económico. De no considerarse, se pueden desaprovechar sus cualidades.

A pesar de que el patrocinio es un formato muy utilizado, y por lo tanto familiar para el espectador, mantiene niveles de recuerdo excelentes: triplican el recuerdo espontáneo de la publicidad convencional y casi doblan su recuerdo asistido. Uno de los elementos que explicaría este mayor nivel de recuerdo, es la valoración positiva por parte de la audiencia hacia los anunciantes vinculados a los programas mediante patrocinios; anunciantes a los que el espectador reconoce como "financiadores" de sus programas favoritos. De esta manera se generan asociaciones positivas y sinergias entre la marca/producto y el programa (Masterson, 2005). Por ello se hace necesario fomentar acuerdos de patrocinio estables y duraderos en el tiempo, que incidan en la adecuada transmisión de valores entre los programas y las marcas. También se encuentra un ámbito de desarrollo en esta tipología trabajando en sus aspectos creativos, adaptando al máximo la creatividad del anunciante a las peculiaridades del programa patrocinado y la cadena que lo emite.

Respecto a los microprogramas, la atractiva simbiosis entre contenido y publicidad estimula el recuerdo publicitario. Sin embargo, en este trabajo, este formato ha obtenido uno de los niveles de recuerdo más bajos entre las nuevas formas publicitarias estudiadas. Ello se puede explicar porque los microprogramas proponen contenidos informativos/publicitarios afines a públicos muy concretos (Reinares y Reinares, 2007). Es un formato que tiene, por tanto, entre sus cualidades, la gran capacidad de segmentación. Por ejemplo, si el lanzamiento de un vehículo todoterreno se integra en el microprograma semanal sobre el estado de las pistas de esquí, se generará un elevado nivel de recuerdo en las personas afines a este deporte, pero más modesto en la audiencia en general. Los microprogramas reducen en gran medida los frenos con 
los que las audiencias se enfrenta a la publicidad, al tener grandes similitudes con los programas convencionales; consiguiendo una gran cercanía y credibilidad entre el público que persiguen. Cabe recordar que los antecedentes teóricos justificarían que los microprogramas se recuerdan más que los spots por su capacidad para entretener a las audiencias al ser percibidos como programas en vez de mensajes estrictamente comerciales. Por ello es una tipología cuya capacidad para actuar sobre el recuerdo dependerá mucho de la creatividad de los contenidos en los que se basa y del estilo de la producción. Pero, sobre todo, de la adecuada investigación previa que analice los contenidos más idóneos para el público objetivo y la coherencia entre el microprograma y el producto/servicio al que se vincula.

Una de las mayores virtudes comunicacionales de las telepromociones externas es permitir vínculos con los personajes y las localizaciones de las series. Como elemento fundamental de su gestión, es imprescindible que la acción publicitaria respete el tono de la serie a la que se vinculan, ya sea drama o comedia. Se debe proteger y mantener la identidad de los actores que participan en la acción, respetando al máximo los roles que interpretan en sus series, de forma que la asociación con el producto no resulte forzada. De otra manera, el impacto puede ser incluso negativo, al percibirse como una utilización comercial del personaje que los espectadores rechazan. Sobre las telepromociones internas, destaca como elemento fundamental de su gestión, la correcta integración en (y con) los contenidos de los programas. Cuando se sitúa al espectador ante comunicaciones artificiosas o forzadas en exceso se genera rechazo en las audiencias (Reinares y Esteve, 2008). De nuevo, se recomienda un profundo análisis previo que certifique la coherencia entre el tono y los contenidos del programa, sus conductores y el producto/servicio al que se vinculan; que determine, en suma, los contenidos más idóneos para el público objetivo buscado.

Cabe destacar las implicaciones operativas de los altos niveles de recuerdo espontáneo, en la línea de los resultados de trabajos como los de Reinares y Reinares (2011) y Martín et al. (2012), generados por las telepromociones internas y externas. Ello permite inferir que se trata de dos formatos especialmente indicados para anunciantes nuevos o menos conocidos; con presupuestos modestos y que necesiten notoriedad a corto plazo, o con objetivos de comunicación tácticos, por ejemplo con promociones puntuales.

Como conclusión final, dada la generalización de oferta de los nuevos formatos entre las diferentes cadenas, se deben considerar diferentes condicionantes, específicos de estos formatos, que incrementarían la complejidad de su gestión operativa y su introducción en un plan de medios.

Los resultados presentados deben valorarse con las limitaciones propias de un trabajo de carácter exploratorio, cuyo origen se encuentra en las necesidades de información de los agentes del medio de televisión y la carencia de investigación académica al respecto. En este sentido, será necesario profundizar en los condicionantes específicos que ocasionan mayor recuerdo de las nuevas formas de publicidad que se describen en este trabajo. De esta manera se podría aportar una mayor 
compresión sobre la influencia de los elementos formales, asociados a cada formato. Factores como la influencia de los presentadores, las asociaciones con los programas, su posición dentro o fuera del bloque publicitario, la duración del formato, o sus elementos creativos, son algunos factores que, en futuros avances de la investigación, deben aislarse en su análisis. En la línea de la discusión de nuestros resultados, un paso obligado posterior se dirigirá hacia la identificación de las variables creativas y relacionadas con las características sociodemográficas de la audiencia que puedan explicar los resultados obtenidos en este trabajo.

Por otra parte, la valoración de la eficacia de publicidad en términos de recuerdo, es una visión parcial de los potenciales efectos de las acciones publicitarias en las audiencias. En este sentido, se debe tener en cuenta que algunas fuentes (Balasubramanian, 1994; Martín et al., 2012) avanzan que la eficacia medida en términos afectivos, puede reportar valores en sentido contrario a los obtenidos en la eficacia en términos cognoscitivos. Por lo tanto, en futuras líneas de investigación será trascendental entender las relaciones entre eficacia cognoscitiva y eficacia afectiva para poder aportar una visión integral de las actuales prácticas publicitarias del medio televisión.

Por último, debido al interés sectorial que pueden tener para los anunciantes estos resultados, parece interesante desarrollar, a partir de una adaptación de la metodología propuesta, nuevos estudios encaminados a analizar el efecto de las formas publicitarias sobre marcas específicas.

\section{Referencias bibliográficas}

AMOS, C.; HOLMES, G.; STRUTTON, D. (2008). "Exploring the relationship between celebrity endorser effects and advertising effectiveness. A quantitative synthesis of effect size". En International Journal of Advertising, vol. 27, n 2 . p.209-234.

ARRAZOLA, M.; HEVIA, J.; REINARES, P.; REINARES, R. (2013). "Do new forms of television advertising occasion better recall than traditional advertising spots?. En International Journal of Advertising, vol. 32, $\mathrm{n}^{\circ}$. 2. p.281-300. doi: 10.2501/IJA-32-2-281-300

BADDELEY, A. (1990). Human Memory: Theory and Practice. Boston: Allyn \& Bacon.

BALASUBRAMANIAN, S.K. (1994). "Beyond Advertising and Publicity: Hybrid Messages and Public Policy Issues". En Journal of Advertising, vol. 23 n 4 4. p.2946. doi: 10.1080/00913367.1943.10673457

BARTEL-SHEEHAN, K.; GUO, A. (2005). "Leaving on a branded jet plane: An exploration of audience attitudes towards product assimilation in television content". En Journal of Current Issues and Research in Advertising, vol.27, $\mathrm{n}^{\circ} 1$. p.79-91. doi: 10.1080/10641734.2005.10505175 
BEERLI, A.; MARTÍN, J.D. (1998). "Memory-Based Advertising Effectiveness Techniques: Recall versus Recognition". En: ZOPOUNIDIS, C. Y PARDALOS, P.M. (Eds.). Managing in Uncertainty: Theory and Practice. Amsterdam: Kluwer Academic Publishers. p.183-201. doi: 10.1007/978-1-4757-2845-3_13

BURKE, R.; SRULL, T.K. (1988). "Competitive Interference and Consumer Memory for Advertising”. En Journal of Consumer Research, vol.15, nº1. p.55-68. doi: $10.1086 / 209145$

CARAT (2005). Quantitative impact of the regulation on TV advertising markets in EU Member States, EEA countries, the new Member States and a number of third party countries. Report 3 18/18. Stages B and C. Disponible en http://ec.europa. eu/avpolicy/docs/library/studies/2003/44_03_re_us.pdf [20/09/2013].

CHOWDHURY, R.; FINN, A.; OLSEN, G. (2007). "Investigating the Simultaneous Presentation of Advertising and Television Programming". En Journal of Advertising, vol.36, n³. p.85-96. doi: 10.2753/JOA0091-3367360306

DAHLÉN, M.; EDENIUS, M. (2007). "When is advertising advertising? Comparing responses to traditional and non- traditional advertising media". En Journal of Current Issues and Research in Advertising, vol. 29, $\mathrm{n}^{\circ} 1$. p.33-42. doi: 10.1080/10641734.2007.10505206

DAWSON, C. (1996). “Television advertising: In need of reinvention?". En International Journal of Advertising, vol.15, $\mathrm{n}^{\circ} 4$. p.302-313. doi: 10.1111/j.02650487.1996.00030.pp.x

DE PELSMACKER, P.; GEUENS, M.; VANDEN BERGH, J. (2005). Marketing Communicatie. Amsterdam: Pearson Education.

EGTA (2008). Egta Practical guide on the EU directive on AMS. Brussels: Association of television and radio sales houses. Egta.

FARRÉ, J.; FERNÁNDEZ, J. (2005). "Non-Conventional Advertising on Full-Service Television". En Quaderns del CAC, vol.22 n mayo-agosto. p.3-18.

FERNÁNDEZ CAVIA, J. (2005). "Non-Conventional Formats and the Transformation of the Television Advertising Business". En Quaderns del CAC, vol.22, $\mathrm{n}^{\circ}$ mayo-agosto. p.33-45.

FURNHAM, A.; BERGLAND, J.; GUNTER, B. (2002). "Memory for television advertisements as a function of advertisement-programme congruity". En Applied Cognitive Psychology, vol.16, $\mathrm{n}^{\circ}$ 5. p.525-545. doi: 10.1002/acp.812

GUPTA, P.B.; GOULD, S.J. (2007). "Recall of Products Placed as Prizes Versus Commercials in Game Shows". En Journal of Current Issues and Research in Advertising, vol.29, no.1. p.43-54. doi: 10.1080/10641734.2007.10505207

HA, L.; MCCANN, K. (2008). "An integrated model of advertising clutter in offline and online media". En International Journal of Advertising, vol.27, n.4 p.569592.

HACKLEY, C.; TIWSAKUL, A. (2006). "Entertainment Marketing and Experiential Consumption". En Journal of Marketing Communications, vol.12, $\mathrm{n}^{\circ} 1$. p.63-75. doi: $10.1080 / 13527260500358608$ 
HETSRONI, A.; ASYA, I. (2002). "A Comparison of values in infomercials and commercials". En Corporate Communications: An International Journal, vol.7, nº 1. p.34-45. doi: 10.1108/13563280210416026

IP NETWORK (2007). Sectorial Survey Television. IP Network.

JEONG, Y.; KIM, Y.; ZHAO, X. (2011). "Competing for consumer memory in television advertising: an empirical examination of the impacts of non-editorial clutter on brand memory in mega-event broadcasts". En International Journal of Advertising, vol.30, no 4. p.617-640. doi: 10.2501/IJA-30-4-617-640

LARDINOIT, T. (1999). "Interaction des parrainages terrain et TV : impact sur deux niveaux de mémorisation". En: Proceedings of the 15th Congress of the French Marketing Association AFM. Strasbourg: Association Française de Marketing. p.585-602.

LEE, M.; FABER, R.J. (2007). "Effects of product placement in on-line games on brand memory: a perspective of the limited-capacity model of attention". En Journal of Advertising, vol.36, nº 4. p.75-90. doi: 10.2753/JOA0091-3367360406

LI, C. (2010). "Primacy effect or recency effect? A long-term memory test of Super Bowl commercials". En Journal of Consumer Behaviour, vol.9, nº 1. p.32-44. doi: 10.1002/cb.291

LORD, K.R.; BURNKRANT, R.E. (1993). "Attention versus distraction: The interactive effect of program involvement and attentional devices on commercial processing". En Journal of Advertising, vol.22, $\mathrm{n}^{\circ} 1$. p.47-60. doi: 10.1080/00913367.1993.10673396

LOWREY, T.M. (2006). "The relation between script readability and commercial memorability". En Journal of Advertising, vol.35, n³. p.7-15. doi: 10.2753/ JOA0091-3367350301

LOWREY, T.M.; SHRUM, L.J.; MCCARTY, J.A. (2005). "The Future of Television Advertising". En: KIMMEL, A.J. (Ed.). Marketing Communication: Emerging Trends and Developments. New York: Oxford University Press. p.113-132.

MARTÍN, J.D.; REINARES, E.; REINARES. P. (2012). “Análisis comparativo de la eficacia publicitaria en televisión: telepromoción versus spot". En Revista Española de Investigación y Marketing, vol.16, n² 2. p.28-47. doi: 10.1016/S11381442(14)60014-0

MASTERSON, R. (2005). "The importance of creative match in television sponsorship". En International Journal of Advertising, vol.24, nº 4. p.505-526.

MCDANIEL, S.R. (1999). "An investigation of match-up effects in sport sponsorship advertising". En Psychology and Marketing, vol.16, n² 2. p.163-184. doi:10.1002/ (SICI) 1520-6793

MEDIA PLANNING GROUP (2007). Eficacia publicitaria de las acciones publicitarias especiales en televisión. Media Planning Group.

MEIR, R.; ARTHUR, D.; TOBIN, J.; MASSINGHAM, C. (1997). "Professional rugby league in Australia: a case study in sponsor awareness". En Cyber-Journal of Sport Marketing, vol.1, no2. p.83-91. 
MOORMAN, M.; NEIJENS, P.; SMIT, E. (2007). "The Effects of Program Involvement on Commercial Exposure and Recall in a Naturalistic Setting". En Journal of Advertising, vol.36, $\mathrm{n}^{\circ} 1$. p.121-137. doi: 10.2753/JOA0091-3367360109

NEWELL, S.J.; HENDERSON, K.V.; WU, B.T. (2001). "The Effects of Pleasure and Arousal on Recall of Advertisements during the Super Bowl". En Psychology and Marketing, vol.18, $\mathrm{n}^{\circ} 11$. p.1135-1153. doi: 10.1002/mar.1047

PIETERS, R.; BIJMOLT, T. (1997). "Consumer Memory for Television Advertising: A Field Study of Duration, Serial Position, and Competition Effects". En Journal of Consumer Research: An Interdisciplinary Quarterly, vol.23, nº 4. p.362-372.

REINARES, P.; REINARES, E. (2003). Fundamentos básicos de la gestión publicitaria en televisión. Madrid: Esic Editorial.

(2007). "Analysis of the contents of the advertising saturation on television". En Esic Market, n'126. p.23-94.

(2011). "The Effectiveness of Next Television Advertising Formats - A RealTime Experiment in Spain”. En Marketing Review St. Gallen, n5. p.56-61. doi: 10.1007/s11621-011-0064-7

REINARES, P.; REINARES, R. (2009). "Managing television sponsorship: a proposal based on its assessment by agents in the Spanish advertising system". En Journal of Sponsorship, vol.2, n³. p.37-48.

REINARES, R.; ESTEVE, A. (2008). “¿Me recuerdas?: variables que influyen en el recuerdo publicitario". En Top Spot, vol.4, primer trimestre. p.42-46.

ROMANIUK, J.; WIGHT, S. (2009). "The influence of brand usage on responses to advertising awareness measures". En International Journal of Market Research, vol.51, no2. p.203-218. doi: 10.2501/s1470785309200426

ROMANIUK, J. (2009). "The Efficacy of Brand-Execution Tactics in TV Advertising, Brand Placements, and Internet Advertising". En Journal of Advertising Research, vol.49, no2. p.143-150. doi: 10.2501/S0021849909090187

ROSS, H.L. (1982). "Recall versus Persuasion: An answer". En Journal of Advertising Research, vol.22, $\mathrm{n}^{\circ}$.1. p.13-17.

ROTHSCHILD, M.; CHURCHILL Jr.,G. (1998). "Recognition versus recall as measures of television commercial forgetting". En Journal of Marketing Research, vol. $25, \mathrm{n}^{\circ} 1 . \mathrm{p} .72-81$.

RUBINSON, J. (2009), "Empirical Evidence of TV Advertising Effectiveness". En Journal of Advertising Research, vol.49, nº2. p.220-226. doi: 10.2501/ S0021849909090321

RUSSELL, C.A. (2002). "Investigating the Effectiveness of Product-Placements in Television Shows: The Role of Modality and Plot Connection Congruence on Brand Memory and Attitude". En Journal of Consumer Research, vol.29, n³. p.306-318. doi: 10.1086/344432

SCHMID, A. (2003). "Are New TV Advertising Formats More Effective?, IP Deutschland and RTL Television. Disponible en http://www.ipbelgium.be/upload/ TV/marketing/formats/effectivesplit/newtvads.pdf[07/09/2013]. 
SCOTT, W. (2005). "Serial position effects in n recall of television commercials". En The Journal of General Psychology, vol.132, n². p.151-164. doi: 10.3200/ GENP.132.2.151-164

SHARP, B.; BEAL, V.; COLLINS, M. (2009). "Television: back to the future". En Journal of Advertising Research, vol.49, n². p.211-219. doi: 10.2501/ S002184990909031X

SMIT, E.; VAN REIJMERSDAL, E.A.; NEIJENS, P. (2009). "Today's practice of brand placement and the industry behind it". En International Journal of Advertising, vol.28, $\mathrm{n}^{\circ} 5$. p.761-782. doi: 10.2501/S0265048709200898

STANTON, J.L.; BURKE, J. (1998). "Comparative effectiveness of executional elements in TV advertising: 15- versus 30-second commercials". En Journal of Advertising Research, vol.38, n6. p.7-14.

TIWSAKUL, R.; HACKLEY, C.; SZMIGIN, I. (2005). "Explicit, Non-integrated Product Placement in British Television Programmes: an exploratory study". En International Journal of Advertising, vol.24, $\mathrm{n}^{\circ} 1$. p.95-111.

TSE, A.C.B.; LEE, R.P.W. (2001). "Zapping behavior during commercial breaks". En Journal of Advertising Research, vol.41, n³. p.25-29.

TUR VIÑES, V.; SEGARRA SAAVEDRA, J. (2013). "Los comienzos del morfing y del SMS en la publicidad televisiva española". En Historia y Comunicación Social, vol.18, no Especial Octubre. p. 555-570. doi:10.5209/rev_HICS.2013. v18.43988

VAKRATSAS, D.; AMBLER, T. (1999). "How Advertising Works: What Do We Really Know?". En Journal of Marketing, vol.63, n.1. p.26-43.

WALLISER, B. (2003). "An international review of sponsorship research: extension and update". En International Journal of Advertising, vol.22, $\mathrm{n}^{\circ} 1$. p.5-40.

WOODS, L. (2008). "The consumer and advertising regulation in the Television Without Frontiers and Audiovisual Media Services Directives". En Journal of Consumer Policy, vol.31, nº 1 . p.63-77. doi: 10.1007/s10603-007-9057-y

\section{Notas}

1 El desarrollo de la investigación en un entorno real de emisiones publicitarias implica un desequilibrio entre la muestra de spots y las FNC.

2 Existen diferencias significativas. Se ha realizado el test de igualdad de medias ( $\mathrm{Z}$ test); Recuerdo espontáneo: 9,3531; Recuerdo asistido: 23,7609.

3 Para efectuar este análisis se contrasta la hipótesis de si la proporción de individuos que recuerdan de forma asistida el anuncio es la misma entre los spots y cada una de la cuatro formas no convencionales. Empleando el estadístico Chi-cuadrado, las diferencias entre los spots y las formas no convencionales es significativa, pues en los cuatro casos se obtiene un p-valor menor de 0,001 .

4 Con respecto al recuerdo espontáneo, empleando el estadístico Chi-cuadrado, las diferencias entre los spots y los patrocinios, telepromociones internas y externas es significativa, en los tres casos se obtiene un p-valor menor de 0.001. Sin embargo las diferencias no son significativas al comparar el recuerdo espontáneo obtenido entre los spots y los micropro- 
gramas ( $\mathrm{p}$-valor $=0,1510)$. Esto puede ser atribuible al reducido número de personas $(10)$ que de forma espontánea recuerdan los microprogramas. 